\title{
UMA AULA DE WALTHER NERNST NO BRASIL
}

\section{Cássius Klay Nascimento ${ }^{\mathrm{a}}$ e João Pedro Braga ${ }^{\mathrm{b}, *}$}

a'Laboratório de Química, Escola Preparatória de Cadetes do Ar, 36205-058 Barbacena - MG, Brasil

'Departamento de Química, Universidade Federal de Minas Gerais, 31270-901 Belo Horizonte - MG, Brasil

Recebido em 02/10/2018; aceito em 18/12/2018; publicado na web em 31/01/2019

\begin{abstract}
WALTHER NERNST LECTURE IN BRAZIL. Walther Hermann Nernst is known to have established the basis for the third law of thermodynamics and also as one of the founders of the modern physical chemistry. For his work in chemical thermodynamics, he was awarded the Nobel Prize in chemistry, in 1920. An important fact about this scientist life, not always realized, was his stay in Brazil, in 1914, for a short period. He went to the city of São Paulo, where he gave a conference at the Faculdade São Bento. He talked about thermodynamics to a very selected audience. The work presented here explores this visit to Brazil, the importance the newspaper gave to this important visitor, and the community present at his lecture. His impression of Brazil was certainly positive, since one of his daughter immigrates to Brazil in 1939. It is hoped the material will add relevant information to the history of chemistry in Brazil.
\end{abstract}

Keywords: Nernst in Brazil; third law of thermodynamics; Faculdade de São Bento.

\section{INTRODUÇÃO}

Em abril de 1914, Walther Hermann Nernst visita a Universidade de La Plata, Argentina, durante seis semanas. ${ }^{1}$ Com essa informação, parece natural a pergunta: é possível que Walther Nernst tenha também visitado o Brasil na sua viagem à Argentina?

O trabalho aqui apresentado é consequência desse questionamento inicial, feito pelos presentes autores. Um fato desconhecido normalmente da comunidade científica é que o físico-químico visitou o Brasil em maio de 1914, onde se encontrou com diversas personalidades da comunidade científica, educacional, cultural e empresarial na cidade de São Paulo e proferiu uma palestra no Colégio e Faculdade de São Bento. O presente artigo busca resgatar fatos da visita desse renomado cientista ao Brasil e o destaque que a imprensa da época deu para este evento.

\section{A PERSONALIDADE DE WALTHER NERNST}

Os fundamentos da terceira lei da termodinâmica foram estabelecidos por Walther Nernst, quando estudava equilíbrio químico. ${ }^{2}$ Apesar de publicado em 1906, o trabalho foi apresentado na Academia de Göttingen, ${ }^{3}$ Alemanha, em 1905. A produção científica recebeu o nome de $O$ novo teorema do calor, e as melhores fontes para entender esse teorema se encontram nos livros que escreveu, um com o mesmo título do trabalho apresentado e outro, um tratado de química teórica. ${ }^{4,5}$

Três de seus alunos de doutorado, Francis Eugen Simon (Simon), Kurt Alfred Georg Mendelssohn (Mendelssohn) e Frederick Alexander Lindemann (mais tarde Lord Cherwell) retratam bem outros aspectos da vida particular e da personalidade de Walther Nernst. ${ }^{6,7}$

Nernst tinha uma grande vitalidade para a pesquisa, trabalhando até perto de sua morte, com quase 80 anos. Estima-se a produção do cientista por volta de 20000 a 25000 páginas, com interesse em várias áreas além da físico-química, incluindo pintura, música, filosofia e interação com a indústria. ${ }^{6} \mathrm{~A}$

*e-mail: jpbraga@ufmg.br personalidade do pesquisador era algo marcante também, como relatada por F. Simon:? era bom trabalhar perto de Nernst, mas não tão perto, por causa da natureza impulsiva de sua personalidade. Pode-se observar no seu livro de Físico-Quimica, ${ }^{8}$ que a terceira lei é citada como minha fórmula, assim como meus estudantes e meu laboratório. ${ }^{6}$ Nas suas aulas, o pesquisador explicava que a quarta lei da termodinâmica não poderia existir. Segundo ele, a primeira lei precisou de três cientistas para ser estabelecida, Mayer, Joule e Helmholtz. A segunda, somente dois cientistas, Carnot e Clausius. A terceira somente um, Nernst. Portanto, nenhum cientista seria necessário para a quarta lei da termodinâmica. ${ }^{6}$

Alguns aspectos da personalidade marcante de Nernst aparecem também nas correspondências trocadas entre Mendelssohn e Lindemann com familiares do cientista. ${ }^{9} 10$

\section{O TRABALHO DE WALTHER NERNST}

Walther Hermann Nernst nasceu em 25 junho de 1864 na cidade de Wąbrzeźno (em alemão Briesen), na Polônia, na época do seu nascimento a cidade pertencia à Prússia.

$\mathrm{O}$ cientista foi um dos fundadores da moderna físicoquímica. Seu trabalho teórico e experimental em química, incluindo sua formulação do teorema do calor, conhecido como a Terceira Lei da Termodinâmica, valeu-lhe o Prêmio Nobel de Química de 1920. O cientista já havia sido indicado ao Nobel em 1911, mas a laureada naquele ano foi Marie Sklodowska Curie. ${ }^{11-13}$

O físico-químico estudou na Universidade de Zurique, na Suíça, na Universidade de Graz, na Áustria, e depois na Alemanha, na Universidade de Berlim, antes de obter seu doutorado, em 1887, na Universidade de Würzburg, também na Alemanha. Após a formatura, ele se tornou assistente de Friedrich Wilhelm Ostwald (1853-1932), que recebeu o Nobel de Química em 1909. A dupla de cientistas, em parceria com Jacobus Henricus van't Hoff (1852-1911) e Svante August Arrhenius (1859-1927), estabeleceu as bases de um novo campo teórico e experimental de pesquisa na química. Pelas suas investigações conjuntas de fenômenos em soluções, em particular o transporte de eletricidade e matéria, esses 
pesquisadores obtiveram importantes descobertas sobre o funcionamento de reações químicas e também estabeleceram o que ficou conhecido como a físico-química moderna. ${ }^{14}$

Nernst dedicou-se ao cálculo do coeficiente de difusão de eletrólitos para soluções infinitamente diluídas e ao estabelecimento de uma relação entre mobilidade iônica, coeficientes de difusão e força eletromotriz eletroquímicas. Em 1889, estabeleceu uma conexão fundamental entre termodinâmica e teoria da solução eletroquímica (desenvolveu a conhecida equação de Nernst). ${ }^{14}$

Devido ao seu trabalho, o pesquisador foi nomeado professor associado da Universidade de Göttingen, em 1891. Nernst publicou um importante livro em 1893; Theoretical chemistry from the standpoint of Avogadro's rule and thermodynamics. ${ }^{5}$

Em 1894, passou a ocupar a cadeira de físico-química no recém-criado Instituto de Físico-Química e Eletroquímica em Göttingen, Alemanha (Figura 1), onde lançou um ambicioso programa de pesquisa em equilíbrio químico, teoria da solução, pressão osmótica e eletroquímica.

Em Göttingen também se dedicou ao desenvolvimento de uma nova lâmpada elétrica. Nernst tinha o objetivo de melhorar o funcionamento da lâmpada incandescente. Descobriu que o óxido de magnésio, que não conduz eletricidade à temperatura ambiente, torna-se um condutor elétrico excelente em altas temperaturas, emitindo uma luz branca brilhante quando empregado como um filamento. Em 1897, ele começou a trabalhar na lâmpada elétrica, pela qual obteve numerosas patentes na Europa e nos Estados Unidos. A lâmpada Nernst foi fabricada por vários anos pela Allgemeine Elektrizitätsgesellschaft (AEG), em Berlim, e milhares destas lâmpadas decoraram um pavilhão alemão especialmente construído na Exposição Internacional de Paris de $1900 .^{15}$

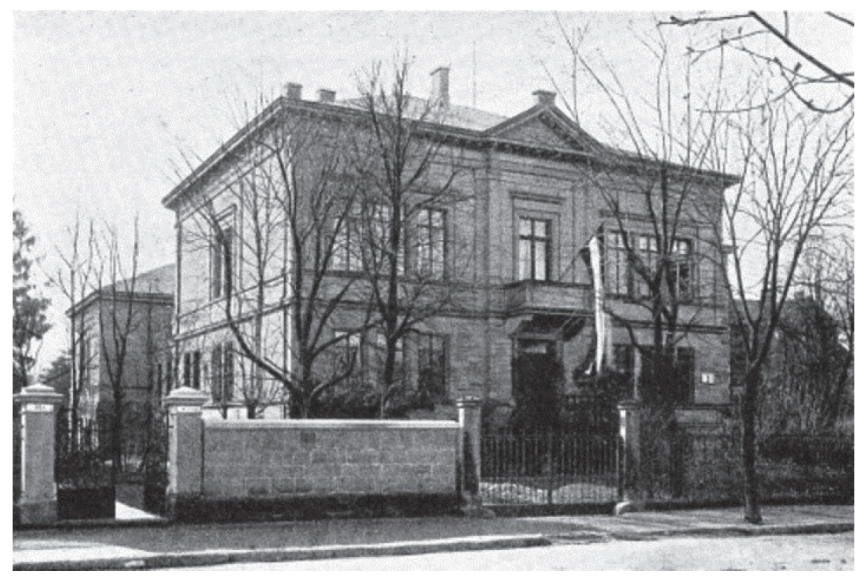

Figura 1. Instituto de Físico-Química de Göttingen foto de Friedrich Dolezalek. (Fonte: Museum of Chemistry at Goettingen)

Em 1905, Nernst foi nomeado professor e diretor do Segundo Instituto de Química da Universidade de Berlim e membro permanente da Academia Prussiana de Ciências. ${ }^{1}$

O cientista foi chefe do departamento de físico-química da Universidade de Berlim, em 1905, reitor da Universidade, em 1921, chefe do Departamento Nacional Alemão de Padrões Físicos, em 1922, e diretor do Instituto de Física Experimental, em 1924. Aposentou em 1933 (Figura 2) e de certa forma se isolou por profundas divergências com Hitler e o partido nazista. ${ }^{14}$

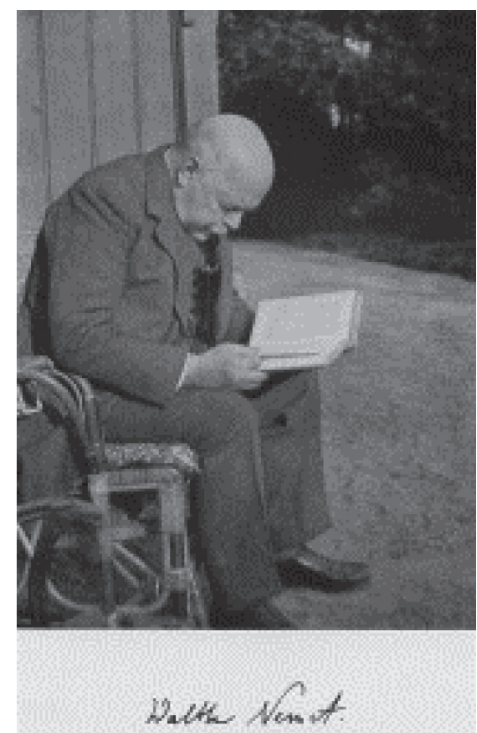

Figura 2. Nernst fotografado por sua filha Angela Hahn, em 1934, no início de sua aposentadoria. (Fonte: Museum of Chemistry at Goettingen)

Walther Hermann Nernst faleceu em 18 de novembro de 1941, aos 77 anos, na cidade de Niwica, atualmente pertencente à Polônia, onde ele tinha uma propriedade rural. O túmulo do cientista e de parte de sua família está na cidade de Göttingen, na Alemanha.

\section{WALTHER NERNST EM SÃO PAULO}

Em maio de 1914, pouco antes do início da Primeira Guerra Mundial, São Paulo experimentava um grande desenvolvimento industrial e econômico, e os cafeicultores viviam seu apogeu. É neste clima que chega Walther Hermann Nernst à cidade. ${ }^{16}$

Após o término de sua estada em La Plata, o pesquisador não retorna imediatamente para Europa. Antes, vem ao Brasil e apresenta uma conferência no Gymnasio de São Bento, na cidade de São Paulo, atualmente o Colégio e a Faculdade de São Bento, fundada pelos monges beneditinos. ${ }^{17}$

Em sua palestra, que teve duração de duas horas e foi proferida em francês, o cientista falou do desenvolvimento da termodinâmica química. ${ }^{17}$ Os principais jornais brasileiros da época, que destacaram a presença de Nernst em São Paulo, serão aqui apresentados. Nos textos em português, optou-se por manter a ortografia da época, observação que se aplica ao restante do artigo.

O Jornal Correio Paulistano, de 22 de maio de 1914 na sua primeira página, deu destaque à visita de Nernst, apresentando o local, horário, tema da palestra e lembrou que o cientista era um ilustre sábio respeitado em todo mundo pelo seu conhecimento e contribuição à ciência. O texto, intitulado Conferência Scientifica, diz:

O notavel scientista alemão, sr. Dr. Walter Nernst, ora de passagem nesta capital, realizou hontem, ás 10 horas, no salão do Gymnasio de S. Bento, a sua anunciada conferencia sobre "O desenvolvimento da Thermo-dynamo-chimica".

O ilustre sábio, que falou em língua franceza, discorreu por espaço de duas horas, sobre o thema proposto, com uma erudição a altura do renome de que gosa no mundo scientifico.

Entre as várias personagens ilustres que participaram da apresentação de Nernst na cidade de São Paulo, ${ }^{17}$ destacam-se as seguintes, que passamos a enumerar: 
Eduardo Augusto Ribeiro Guimarães (1860-1931), formado em Medicina. Radicou-se em Campinas, onde se dedicou à política, à medicina, à agricultura. Mais tarde mudou-se para a capital paulista e foi o primeiro reitor da antiga Universidade de São Paulo, não tem relação com a USP. ${ }^{18}$ A Universidade, criada em 1911, era uma escola particular, tinha como proposta a formação de alunos nos diferentes níveis de ensino. Fundou, entre outros estabelecimentos, a primeira Faculdade de Medicina de São Paulo e o Hospital de Caridade do Braz (Atualmente Hospital Santa Virgínia). Formou, na área da Saúde, alunos nos cursos de odontologia, farmácia e medicina, até a antiga Universidade de São Paulo ser fechada em 1918.

Antônio Francisco de Paula Souza (1883-1917), engenheiro, formado em Karlsruhe (na atual Alemanha). Foi o diretor da Escola Politécnica de São Paulo e é o patrono da engenharia paulistana.

Reynaldo Porchat (1868-1953) foi o primeiro reitor da atual Universidade de São Paulo (USP), uma instituição pública. Nomeado diretor da Faculdade de Direito em 1930, em 1934 foi escolhido reitor da USP. Foi também nomeado membro do Tribunal Regional de Justiça Eleitoral.

Carlos José de Arruda Botelho (1855-1942), médico urologista, formado na França. Tornou-se um dos mais brilhantes e reconhecidos cirurgiões de São Paulo. Ajudou a fundar a Policlínica de São Paulo, e foi presidente da Academia de Medicina de São Paulo entre 1896 a 1917. Criou a Agência Nacional de Colonização, em 1907, e, em 1908, assinou um contrato pela chegada do navio Kasato Maru, tornando-se, no Brasil, o introdutor dos primeiros imigrantes vindos do Japão no país.

Geraldo Horácio de Paula Souza (1889-1951) foi o fundador do Instituto de Higiene e da Faculdade de Higiene e Saúde Pública (núcleos que deram origem à atual Faculdade de Saúde Pública da Universidade de São Paulo).

Affonso d'Escragnolle Taunay (1876-1958), Affonso de Taunay, apesar de ter se formado em engenharia pela Politécnica de São Paulo, era historiador. Organizou e divulgou as obras de seu pai, Alfredo d'Escragnolle Taunay, Visconde de Taunay (1843-1889). Marcou a historiografia brasileira na primeira metade do século XX, principalmente por sua atuação como diretor do Museu Paulista, entre 1917 a 1945.

Dom Miguel Kruse (1864- 1929), em 1900, assumiu a direção do Mosteiro de São Bento em São Paulo. Fundou, em 1903, o Colégio de São Bento e logo após, em 1908, fundou também a faculdade de Filosofia, que seria a primeira do Brasil.

Dom Amaro van Emelen (1863-1943) reitor do Colégio de São Bento, em São Paulo, durante o período de 1905 e 1906, e também, 1909 e 1910.

Raul Briquet (1887-1953) ginecologista e obstetra. Suas publicações, a partir de 1914, propiciaram a criação de uma postura científica, conhecida como Escola Briquet de Ginecologia. Participou da criação da Sociedade Brasileira de Psicanálise, primeira entidade associativa dos psicanalistas brasileiros, tendo ocupado o cargo de vice-presidente. ${ }^{19}$

Estavam presentes também várias outras personalidades paulistanas, além de alunos da Escola Politécnica de São Paulo, da Faculdade de Medicina e Cirurgia de São Paulo, do Mackenzie College, vinculado à Universidade de Nova York (atualmente é a Universidade Presbiteriana Mackenzie).

Com mais detalhes sobre as pessoas presentes à aula de Nernst, descreve o Correio Paulistano na sua primeira página:

Entre as pessoas, que assistiram à brilhante conferência do sr, dr. Walther Nernst, achavam-se os srs. Dr. Eduardo
Guimarães, reitor da Universidade de S. Paulo; dr. Paula Sousa, diretor da escola Polytechnica; dr. Eugen Will, consul da Allemanha; dr. Reynaldo Porchat, lente da Faculdade de Direito; dr. Carlos Botelho, monsenhor dr. Benedicto de Sousa, dr. Geraldo de Paula Souza, padre dr. Luiz Cavalcante, dr. Magalhães Gomes, dr. Affonso Taunay, dr. Gama e silva, dr. Carlos de Moraes Andrade, dr. Rodrigo Claudio da Silva, d. Miguel Kruse, d. Amaro van Emelem, dr. Armando Rodrigues, dr. Briquet, dr. Antonio Pompeu de Camargo, além de muitos alumnos da Escola Polytechnica, Faculdade de Medicina e Mackenzie College.

Outros importantes jornais do Brasil destacaram também a presença do cientista no país como o Gazeta de Notícias, ${ }^{20}$ o Jornal do Brasil, ${ }^{21}$ o Jornal do Commércio, ${ }^{22}$ o O Paiz ${ }^{23}$ e o O Século. ${ }^{24}$ São apresentadas abaixo alguns trechos de jornais que lembram a visita, como no Jornal Gazeta de Notícias, 21 de maio de 1914, que destaca que o importante cientista estava em São Paulo e apresentaria uma conferência sobre uma ciência em franco desenvolvimento. Segue descrito o texto como no original do Gazeta de Notícias:

O Prof. Nernst em S. Paulo. Conferencia sobre o "Desenvolvimento da Thermo-Dynamica e Chimica".

S. PAULO, 20 (A. A.) - Amanhã, no salão do Gymnasio S. Bento, o sábio chimico Nernst, lente da Universidade de Berlim e de passagem por esta capital procedente do Rio da Prata, realisará uma conferencia sobre o "Desenvolvimento moderno da Thermo-dynamica em Chimica.”.

No mesmo sentido do Gazeta de Notícias o Jornal do Brasil de 22 de maio de 1914, indica também na página 9, o tema da conferência que seria apresentada pelo cientista e sua Instituição na qual ele estava ligado em termos acadêmicos como apresentado no texto que se segue:

- Amanhã, no salão do Gymnasio de S. Bento, o sábio chimico Nernst, lente da Universidade de Berlim e de passagem por esta capital, procedente do Rio da Prata, realizará uma conferencia sobre o "Desenvolvimento moderno da Thermo-dynamica em Chimica.

O Jornal do Commercio de 23 de maio de 1914 na página 6 complementa o texto de outros jornais com uma informação sobre quem acompanhava o físico-químico. O Jornal do Comércio confirma que a esposa de Nernst, Emma Lohmeyer Nernst (1871-1949), também acompanhou a visita a São Paulo. Apesar de não indicar o tempo que o ilustre visitante permaneceu, fala que a visita demoraria alguns dias, e também apresenta as ligações acadêmicas do cientista. $\mathrm{O}$ trecho do Jornal dizia:

Acha-se em $S$. Paulo e passará por esta capital onde se demorará alguns dias, o professor Conselheiro Nernst, uma das maiores mentalidades da Allemanha.

O ilustre viajante é professor cathedratico da Universidade de Berlim, membro das academias de sciencias de Berlim e Göttingen e Erlangen Professor de physico-chimica da Universidade de Berlim. Esse scientista viaja com sua senhora.

A presença de Nernst, no atual Colégio e Faculdade de São Bento, e o tema de sua palestra, foram lembrados também por outro importante jornal brasileiro da época. O Paiz, em 21 de Maio de 1914, página 5 noticiava:

S. Paulo, 20. - Amanhã, no salão do Gymnasio S. Bento, o sabio chimico Nernst, lente da Universidade de Berlim, e de passagem por esta capital, procedente do rio da Prata, realizará uma conferencia sobre o Desenvolvimento moderno da thermo-dynamica em chimica.

O jornal O Seculo, de 23 de maio de 1914, na primeira 
página, ainda de forma breve, informa sobre a presença do ilustre visitante, que estava acompanhado de sua esposa. $\mathrm{O}$ pequeno trecho o periódico lembrava:

Encontra-se actuamente em São Paulo, com s. exma senhora, o conselheiro Nernst, profesessor cathedratico da Universidade de Berlim.

Os jornais que destacaram a visita de Walther Nernst, em São Paulo, estavam entre os principais e mais respeitados veículos de comunicação social do Brasil no início do século XX.

\section{FAMÍLIA NO BRASIL}

Nernst teve cinco filhos: Hildegard Elektra Cahn, Angela Hahn, Rudolf Nernst, Gustav Nernst e Edith von Zanthier. Durante a Segunda Guerra Mundial, devido à perseguição crescente, na Alemanha pelos nazistas, duas filhas do cientista foram obrigadas a abandonar seu país natal. Hilde foi para Londres e Angela emigrou, com o marido Albert Hahn e sua filha Ursula Hahn Shears, em 1939, para o Brasil. ${ }^{25,26}$

Angela esteve com seu pai pela última vez um pouco antes da mudança para continente sul-americano. O consulado do Brasil, em Berlim, emitiu documento para Angela viajar, e nele constava que a filha do cientista apresentou um passaporte expedido pela Polícia de Berlim, com data de 30 de julho de 1937. A autorização consular para Angela entrar no nosso país tem data de 5 de julho de 1939. O registro informa que a filha de Nernst não tinha profissão definida, era nascida na cidade de Göttingen e era de nacionalidade alemã, com residência em Berlim.

Após o fim da Segunda Guerra Mundial, em 1946, a esposa de Nernst, Emma Lohmeyer, havia perdido todos os seus bens. Mesmo estando em área que ficou sob dominação russa, conseguiu autorização para sair da Alemanha e ir morar com sua filha Hilde, na Inglaterra. A transferência de Emma para a Inglaterra teve a ajuda fundamental do físico Frederick Lindemann, Lord Cherwell (1886-1957) como registrado em correspondências entre ele e Emma, ${ }^{9}$ que estão sob responsabilidade da Nuffield College Library da Universidade de Oxford.

Em 1947 Lord Cherwell auxiliaria mais uma vez de forma marcante a família Nernst, ajudando a filha mais nova, Angela, a viajar do Brasil até a Inglaterra para visitar sua mãe. ${ }^{9}$ A esposa do cientista, Emma, morreu em 1949, dois anos depois da visita que Angela lhe fez. Alguns anos após a morte de sua mãe, Angela voltou para a Europa e lá permaneceu. ${ }^{9}$

\section{CONCLUSÃO}

Um dos pais da Terceira Lei da Termodinâmica, da físicoquímica moderna e Prêmio Nobel de Química em 1920, o renomado cientista Walther Hermann Nernst, esteve no Brasil em 1914, na cidade de São Paulo. Apresentou seu trabalho a importantes nomes da física, da química, da saúde, da educação e da filosofia.

Devido à importância da visita do ilustre cientista, os principais jornais brasileiros da década de 1910 destacaram a presença de Nernst em São Paulo.

O cientista com sua capacidade científica e sua forte rejeição ao pensamento nazista certamente deixou marcas importantes naqueles que se abriram aos seus ensinamentos.
Coincidentemente a história da família Nersnt passou pelo Brasil antes, durante e após a Segunda Grande Guerra. O Brasil abrigou mais tarde e minimizou o sofrimento, devido à perseguição aos judeus, do seu genro Albert, de sua filha Angela e de sua neta Ursula durante alguns anos.

\section{AGRADECIMENTOS}

Os autores gostariam de agradecer ao CNPq pela ajuda financeira, ao Nuffield College e à Bodleian Library da Universidade de Oxford, Inglaterra, por permitirem o acesso dos autores às correspondências entre a família de Walther Nernst com Kurt Alfred Georg Mendelssohn e Lord Cherwell. Agradecimentos ao professor Ulrich Schmitt, do Museum of Chemistry at Goettingen, Alemanha, pela permissão do uso das fotografias.

\section{REFERÊNCIAS}

1. Jost, W.; Ber. Bunsenges. Phys. Chem. 1964, 6, 525.

2. Braga, J. P.; Físico-química: Aspectos Moleculares e Fenomenológicos, Ed. UFV: Viçosa, 2002.

3. Bartel, H. G.; Huebener, R. P.; Walther Nernst: Pioneer Of Physics, And Of Chemistry, World Scientific: Singapura, 2007.

4. Nernst, W. H.; The New Heat Theorem: Its foundations in theory and experiment, Methuen \& Co: London, 1926.

5. Nernst, W. H.; Theoretical chemistry from the standpoint of Avogadro's rule and thermodynamics, MacMillan and Co.: London, 1904.

6. Mendelssohn, K. A. G.; The world of Walther Nernst: the rise and fall of German science 1864-1941, University of Pittsburgh Press: Pittsburgh, 1973.

7. Simon, F. E.; The Third Law of Thermodynamics, An Historical Survey, Physical Society: London, 1956.

8. Nernst, W. H.; Experimental and Theoretical Applications of Thermodynamics to Chemistry, Yale University Press: New Haven, 1907.

9. Nernst, E. L. [Cartas] 1946-49 Lindemann, F. A. Inglaterra. Oxford University: Nuffield College Library.

10. Hahn, A. [Cartas] 1967-74 Alemanha. Mendelssohn, K. A. G. Inglaterra. Oxford University: Bodleian Library, Special Collections.

11. Braga, J. P.; Nascimento, C. K.; A Visita de Marie Curie ao Brasil, Livraria da Física: São Paulo, 2017.

12. Nascimento, C. K.; Braga, J. P.; Nature 2017, 551, 440.

13. Braga, J. P.; Nascimento, C. K. Maria Skłodowska-Curie w Brazylii; Polish Academy of Sciences/Polish Chemical Society: Gliwice, 2017.

14. Coffey, P.; Cathedrals of Science: The Personalities and Rivalries That Made Modern Chemistry, Oxford University Press: New York, 2008.

15. Ortiz, E. L.; Rev. Bras. Hist. Ciênc. 2009, 2, 40.

16. Ferreira, A. C.; Mahl, M. L.; Letras e identidade: São Paulo no século XX, capital e interior, Annablume: São Paulo, 2008.

17. Correio Paulistano, 1914, 18261, 1.

18. Mott, M. L.; Duarte, I. G.; Gomes, M. T.; Cad. Hist. Ciênc. 2007, 3, 37.

19. Briquet, R.; Psicol. Cienc. Prof., 2005, 25, 168.

20. Gazeta de Notícias, 1914, 140, 3.

21. Jornal do Brasil, 1914, 142, 9.

22. Jornal do Commercio, 1914, 141, 6.

23. O Paiz, 1914, 10818, 5.

24. O Seculo, 1914, 2383, 1.

25. Jornal do Commercio, 1940, 98, 5.

26. Jornal do Commercio, 1940, 235, 8 